\title{
The effect of ambient temperature on locomotor activity patterns in reproductive and non-reproductive female Damaraland mole-rats
}

\author{
M.K. Oosthuizen* and N.C. Bennett
}

Department of Zoology \& Entomology, University of Pretoria, Private Bag X20, 0028, South Africa

\section{Corresponding author:}

Maria Oosthuizen

Dept. Zoology and Entomology

University of Pretoria

Private Bag X20

Hatfield

0028 South Africa

e-mail: moosthuizen@zoology.up.ac.za

\begin{abstract}
The subterranean niche is a specialized environment that presents its inhabitants with a unique set of microclimatic conditions. African mole-rats are strictly subterranean and exhibit a continuum of sociality ranging from solitary to highly social. Colonies of the Damaraland mole-rat comprise of a dominant breeding or reproductive female and one or two male consorts and a number of subordinate and non-reproductive individuals of both sexes. In this study we investigated the locomotor activity patterns of reproductive and non-reproductive female Damaraland mole-rats with the intention to investigate daily timing of activity and activity levels with changes in ambient temperature, and also whether activity would differ between the reproductive castes at different ambient temperatures.
\end{abstract}


The Damaraland mole-rats displayed predominantly nocturnal activity at all three temperatures, and the levels of activity were different for all three temperatures tested. Molerats exhibited the lowest levels of activity at $30^{\circ} \mathrm{C}$, they are most active at $25^{\circ} \mathrm{C}$ while they display intermediate levels of activity at $20^{\circ} \mathrm{C}$. Despite exhibiting the majority of their activity during the night, non-reproductive females display significantly more day time activity compared to the reproductive females at all three temperatures. Nocturnal activity is comparable between reproductive and non-reproductive animals at $20{ }^{\circ} \mathrm{C}$ and $25{ }^{\circ} \mathrm{C}$, but not at $30^{\circ} \mathrm{C}$.

Daily locomotor activity rhythms of the Damaraland mole-rats appear to be relatively flexible and respond to comparatively small changes in ambient temperatures. Differences in daily activity between reproductive and non-reproductive animals may emphasize the existence of physiological and morphological castes in the Damaraland mole-rat.

Keywords: ambient temperature, Damaraland mole-rat, Fukomys damarensis, locomotor activity, non-reproductive, reproductive,

\section{Introduction}

A large number of mammalian species have evolved to utilize the subterranean ecotope (Nevo, 1979). Fossorial mammals range from species that simply shelter in burrows but forage aboveground to strictly subterranean species that possess complex labyrinths of tunnels (Ellerman, 1956). The subterranean niche provides a unique and challenging environment (Šumbera et al., 2004) that is typically low in primary productivity (Nevo, 1979) and at the same time burrowing is energetically very costly (Lovegrove, 1989; Vleck, 1979; Zelova et al., 2010). Typically sealed tunnel systems are characterized by high relative humidities and limited ventilation (Bennett \& Faulkes, 2000; Marhold \& Nagel, 1995; 
Šmbera et al., 2004). Nevertheless subterranean tunnel systems are considered to possess relatively stable and predictable microenvironments that have a low predation risk and daily environmental fluctuations such as temperature changes are minimal even at relatively shallow depths when compared to those above ground (Nevo, 1979).

African mole-rats (family Bathyergidae) are strictly subterranean and construct permanent, yet dynamic tunnel systems where they spend their entire lives (Bennett et al., 2000). The basic tunnel structure for all species is similar, with numerous superficial burrows for foraging which may be only a few centimetres from the surface, while deeper tunnels of up to 1.5-2 metres link to nests and boltholes (Bennett, Jarvis \& Davies, 1988b; Herbst \& Bennett, 2006; Jarvis \& Sale, 1971). Although fluctuations in burrow temperatures are substantially buffered compared to aboveground temperatures (Roper et al., 2001), the actual tunnel temperatures are dependent on the depth of the tunnels and also show seasonal variation (Bennett et al., 1988b; Streicher et al., 2011). Foraging tunnels situated close to the surface show larger temperature fluctuations compared to the deeper tunnels, and are warmer than the deeper tunnels during the summer whereas the opposite is true for the winter (Bennett et al., 1988b; Herbst, Jarvis \& Bennett, 2004; Lovegrove \& Knight-Eloff, 1988; Streicher et al., 2011).

Mole-rats are physiologically well adapted towards their lifestyle (Bennett et al., 2000), exhibiting substantially lower body temperatures and metabolic rates as well as higher thermal conductances when compared to surface dwelling rodents (Bennett, 2009; Bennett et al., 1994; Bennett, Cotterill \& Spinks, 1996; Bennett, Jarvis \& Cotterill, 1993a; Broekman et al., 2006; Lovegrove, 1987; Streicher et al., 2011). Nonetheless, a subterranean existence and the associated foraging is not only substantially more costly than aboveground living (Lovegrove, 1989; Vleck, 1979; Zelova et al., 2010), but generates a significant amount of metabolic heat (Bozinovic, Carter \& Ebensperger, 2005; Luna \& Antinuchi, 2007). In a 
sealed tunnel system with a high humidity, heat dissipation may pose a considerable problem for the inhabitants and animals are at risk of overheating (Bozinovic et al., 2005; Luna et al., 2007; McNab, 1966).

The majority of subterranean species are solitary (Nevo, 1979) but African mole-rats are exceptional in that sociality has arisen in at least three genera of bathyergids. Species from this family display a continuum of sociality, ranging from strictly solitary to highly social or eusocial. The Damaraland mole-rat is a eusocial species with each colony possessing a single breeding female (queen) that is responsible for procreation, all members of the colony cooperatively care for the young and an overlap in litters is present in colonies (Bennett \& Jarvis, 1988a; Jarvis, 1981). Female subordinate mole-rats in the colony are reproductively suppressed by the dominant queen (Bennett, 1994; Bennett et al., 1993b). In Damaraland mole-rats suppression of reproduction is physiological, such that subordinate females are anovulatory (Bennett et al., 1993b; Molteno \& Bennett, 2000). In addition to the reproductive division of labour in the colony, non-breeding animals perform a larger proportion of the foraging and burrow maintenance activities in the tunnel system (Barnett et al., 2003; Bennett et al., 1988a).

Damaraland mole-rats occur in semi-arid areas in the northern parts of South Africa into Namibia and Botswana (Skinner \& Chimimba, 2005). Average temperatures in shallow foraging tunnels (at bulb and tuber levels) are around $31^{\circ} \mathrm{C}$ in summer, while they can have mean temperatures as low as $17^{\circ} \mathrm{C}$ during winter (Streicher et al., 2011).

We investigated the effect of ambient temperature on the daily locomotor activity rhythms of Damaraland mole-rats over a range of temperatures that this species would typically encounter in the more superficial foraging tunnels. In particular we were interested in whether the animals would maintain their preferred times of activity with changes in the ambient temperature, since ambient temperatures below the thermoneutral zone of animals 
may cause phase shifts in activity (Van der Vinne et al., 2014). Many species alter their activity levels with changes in ambient temperatures (Gordon, 1993) therefore we investigated this possibility in the Damaraland mole-rat, and given the division of labour in this species (Bennett et al., 1988a) we considered whether the levels of activity would differ between reproductive and non-reproductive Damaraland mole-rat females over the three defined ambient temperatures.

\section{Material and Methods}

Ethics statement

Trapping and experimental procedures were approved by the Animal Ethics committee of the University of Pretoria (Ref. EC013-09). Collection and export permits were obtained from the Northern Cape nature conservation authorities (Permit Numbers Fauna 464/2009, Fauna465/2009) and an import permit from Gauteng Nature conservation (Permit number CPB6-000769).

\section{Trapping and housing}

All experimental animals were captured in October 2009 using modified Hickman live traps baited with sweet potato. Seven dominant, reproductive (mean body mass $=129.1 \pm 8.5 \mathrm{~g}$ ) and 8 subordinate, non-reproductive (mean body mass $=117.8 \pm 7.6 \mathrm{~g}$ ) female Damaraland molerats were collected in the Blackrock area, Northern Cape, South Africa (2711'58.88'S; $\left.22^{\circ} 57^{\prime} 05.82^{\prime \prime} \mathrm{E}\right)$. Freshly captured mole-rats were transported back to Pretoria where they were housed individually in polyurethane cages $(60 \times 30 \times 30 \mathrm{~cm})$ lined with wood shavings and provided with a nest box and tissue paper as nesting material. Animals were fed $a d$ libitum on sweet potatoes, gem squash and carrots. The wellbeing of the animals was monitored during feeding times. 
Reproductive females were identified by the presence of nipples and a perforate vagina. Nonreproductive females were defined as large females that were not reproductively active at the commencement of the experiment, and since the animals were housed individually, remained so for the duration of the experiment.

Animals were acclimatised in a temperature and light controlled room for two weeks before experimental procedures commenced. Locomotor activity patterns were recorded with infrared motion detectors (Quest PIR internal passive infrared detector; Elite security products (ESP), Electronic lines, UK). These sensors were attached to the top of each cage to detect movement in any area of the cage. Collective activity counts were recorded once per minute and the activity measurements were captured by the program Vital View on a computer system (Vital ViewTM, Minimitter Co., Inc., Sunriver, OR, USA; www.minimitter.com). The study animals were subjected to a 12L:12D light cycle (light: 07h00-19h00) at three different ambient temperatures $\left(20^{\circ} \mathrm{C}, 25^{\circ} \mathrm{C}\right.$ and $\left.30^{\circ} \mathrm{C}\right)$ for approximately one month at each temperature.

\section{Data analyses}

Activity data were depicted as double plotted actograms with Actiview Biological Rhythm Analyses 1.2 software (Minimitter Co., Inc., Sunriver, OR, USA; www.minimitter.com) to visualise activity patterns. Activity counts were summed to obtain hourly values for each day for every animal. Statistical analyses were performed using IBM SPSS Statistics 21.0 (SPSS Inc, Chicago, Illinois, USA). Data were not normally distributed therefore the differences between the reproductive and non-reproductive female Damaraland mole-rats exposed to three different temperatures were assessed with a generalized linear mixed model (GLMM). The hourly activity was used as the dependent variable assuming a Gamma distribution and a log-link function with different days as a repeated measure. We included breeding status, 
time of day (day/night) and temperature as factors in the model. All two and three-way interactions were incorporated, and the least significant difference (LSD) was employed for post hoc comparisons. The significance level was maintained at 0.05 .

\section{Results}

At all three temperatures tested, reproductive and non-reproductive animals exhibited significantly higher amounts of activity during the night compared to during the day (NRF: $20^{\circ} \mathrm{C}, \mathrm{F}=78.3, \mathrm{df}_{1}=1, \mathrm{df}_{2}=18,847, \mathrm{p}<0.001 ; 25^{\circ} \mathrm{C}, \mathrm{F}=166.81, \mathrm{df}_{1}=1, \mathrm{df}_{2}=18,847, \mathrm{p}<0.001 ;$ $30^{\circ} \mathrm{C}, \mathrm{F}=67.91, \mathrm{df}_{1}=1, \mathrm{df}_{2}=18,847, \mathrm{p}<0.001 ; \mathrm{RF}: 20^{\circ} \mathrm{C}, \mathrm{F}=190.74, \mathrm{df}_{1}=1, \mathrm{df}_{2}=18,847$, $\mathrm{p}<0.001 ; 25^{\circ} \mathrm{C}, \mathrm{F}=213.48, \mathrm{df}_{1}=1, \mathrm{df}_{2}=18,847, \mathrm{p}<0.001 ; 30^{\circ} \mathrm{C}, \mathrm{F}=146.3191, \mathrm{df}_{1}=1$, $\mathrm{df}_{2}=18,847, \mathrm{p}<0.001$, Table 1, Figure 1).

Table 1: Total daily- and separate dark and light locomotor activity counts (mean \pm SE) for reproductive and non-reproductive Damaraland females at each of the three ambient temperature tested in this study. RF = reproductive female, $\mathrm{NRF}=$ non-reproductive female .

\begin{tabular}{|c|c|c|c|c|}
\hline Group & Temp & Mean \pm SE & Light phase & Mean \pm SE \\
\hline \multirow{6}{*}{ NRF } & \multirow[t]{2}{*}{$20^{\circ} \mathrm{C}$} & \multirow[t]{2}{*}{$202.9 \pm 12.7$} & Light & $156.6 \pm 10.6$ \\
\hline & & & Dark & $262.9 \pm 17.5$ \\
\hline & \multirow{2}{*}{$25^{\circ} \mathrm{C}$} & \multirow{2}{*}{$263.8 \pm 15.5$} & Light & $179.2 \pm 11.2$ \\
\hline & & & Dark & $388.4 \pm 23.8$ \\
\hline & \multirow[t]{2}{*}{$30^{\circ} \mathrm{C}$} & \multirow[t]{2}{*}{$148.8 \pm 9.6$} & Light & $114.4 \pm 8.1$ \\
\hline & & & Dark & $193.7 \pm 13.4$ \\
\hline \multirow{6}{*}{ RF } & \multirow[t]{2}{*}{$20^{\circ} \mathrm{C}$} & \multirow[t]{2}{*}{$84.4 \pm 5.7$} & Light & $28.6 \pm 2.1$ \\
\hline & & & Dark & $248.9 \pm 17.4$ \\
\hline & \multirow[t]{2}{*}{$25^{\circ} \mathrm{C}$} & \multirow[t]{2}{*}{$193.5 \pm 12.3$} & Light & $83.8 \pm 5.8$ \\
\hline & & & Dark & $446.6 \pm 29.2$ \\
\hline & \multirow[t]{2}{*}{$30^{\circ} \mathrm{C}$} & \multirow[t]{2}{*}{$82.9 \pm 5.7$} & Light & $43.7 \pm 3.3$ \\
\hline & & & Dark & $157.5 \pm 11.5$ \\
\hline
\end{tabular}


Figure 1: Mean activity counts of reproductive and non-reproductive Damaraland mole-rat females at the three different ambient temperatures over 24 hours. Dark periods were from $19 \mathrm{~h} 00$ to $07 \mathrm{~h} 00$, and are indicated with black bars on the $\mathrm{x}$-axis. $\mathrm{RF}=$ reproductive female, $\mathrm{NRF}=$ non-reproductive female.

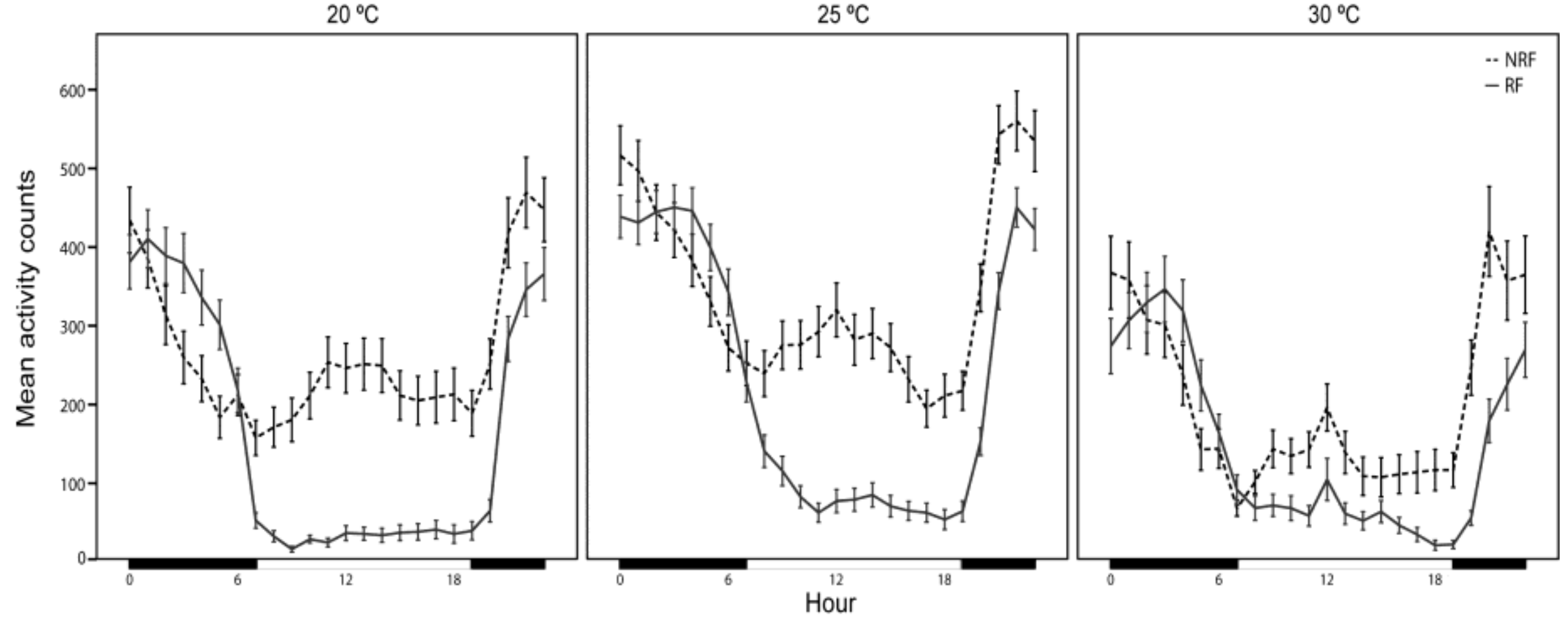


Mole-rats displayed the highest amounts of activity at $25^{\circ} \mathrm{C}$ and the lowest activity levels at $30^{\circ} \mathrm{C}$. In non-reproductive animals, the activity levels differed significantly between all three temperatures tested $\left(\mathrm{F}=71.24, \mathrm{df}_{1}=1, \mathrm{df}_{2}=18,847, \mathrm{p}<0.001\right)$, and whereas activity levels were also different for the respective temperatures in reproductive animals $\left(\mathrm{F}=90.48, \mathrm{df}_{1}=1\right.$, $\left.\mathrm{df}_{2}=18,847, \mathrm{p}<0.001\right)$, it did not differ significantly between $20^{\circ} \mathrm{C}$ and $30^{\circ} \mathrm{C}(\mathrm{p}=0.660$, Table 1, Figure 1).

Reproductive Damaraland females were found to be significantly less active when compared to non-reproductive Damaraland females at each of the individual temperatures tested $\left(20^{\circ} \mathrm{C}\right.$, $\mathrm{F}=72.92, \mathrm{df}_{1}=1, \mathrm{df}_{2}=18,847, \mathrm{p}<0.001 ; 25^{\circ} \mathrm{C}, \mathrm{F}=12.68, \mathrm{df}_{1}=1, \mathrm{df}_{2}=18,847, \mathrm{p}<0.001 ; 30^{\circ} \mathrm{C}, \mathrm{F}$ $=34.94, \mathrm{df}_{1}=1, \mathrm{df}_{2}=18,847, \mathrm{p}<0.001$; Table 1). However, upon further unravelling of the results, it became evident that reproductive females were significantly less active than nonreproductive females during the day at all three temperatures $\left(20^{\circ} \mathrm{C}, \mathrm{F}=139.63, \mathrm{df}_{1}=1\right.$, $\mathrm{df}_{2}=18,847, \mathrm{p}<0.001 ; 25^{\circ} \mathrm{C}, \mathrm{F}=57.48, \mathrm{df}_{1}=1, \mathrm{df}_{2}=18,847, \mathrm{p}<0.001 ; 30^{\circ} \mathrm{C}, \mathrm{F}=65.21, \mathrm{df}_{1}=1$, $\left.\mathrm{df}_{2}=18,847, \mathrm{p}<0.001\right)$. However at night, activity for reproductive and non-reproductive females were comparable at $20^{\circ} \mathrm{C}\left(\mathrm{F}=0.32, \mathrm{df}_{1}=1, \mathrm{df}_{2}=18,847, \mathrm{p}=0.57\right)$ and $25^{\circ} \mathrm{C}(\mathrm{F}=2.40$, $\left.\mathrm{df}_{1}=1, \mathrm{df}_{2}=18,847, \mathrm{p}=0.12\right)$, but not at $30^{\circ} \mathrm{C}\left(\mathrm{F}=4.25, \mathrm{df}_{1}=1, \mathrm{df}_{2}=18,847, \mathrm{p}=0.039\right.$, Table 1 , Figure 1).

\section{Discussion}

\section{Active time and variability}

The temporal activity distribution of the Damaraland mole-rat appears to be slightly ambiguous, with varying results. In the current study, both the reproductive and nonreproductive female mole-rats were found to exhibit more activity during the night. In contrast, a previous study of our group showed Damaraland mole-rats to be primarily diurnal, although some animals displayed nocturnal activity (Oosthuizen, Cooper \& Bennett, 
2003). Lovegrove and colleagues (1993) recorded activity in small colonies of animals in the laboratory, they found the animals to exhibit overall more activity during the day, however a prominent bout of nocturnal activity was also present at the beginning of the dark period where activity peaks were higher than that occurring during the day (Lovegrove, Heldmaier \& Ruf, 1993).

Many species are not rigidly restricted to being diurnal or nocturnal (Mrosovsky \& Hattar, 2005). Temporal niche selection of species is dependent on a variety of external and internal factors that in essence creates a gradient of diurnality between, as well as within species (Refinetti, 2008). Numerous examples exist of species containing both diurnal and nocturnal individuals (Blanchong et al., 1999; Hart et al., 2004; Johnson \& Zucker, 1983; Oosthuizen et al., 2003; Refinetti, 2006; Vasicek et al., 2005), as well as individuals of the same species spontaneously switching between diurnal and nocturnal activity (Mrosovsky et al., 2005; Oosthuizen et al., 2003; Refinetti, 2006). Circadian rhythmicity of locomotor activity patterns is coordinated by an endogenous oscillator in the suprachiasmatic nucleus (SCN) of the hypothalamus (Daan \& Pittendrigh, 1976). The temporal and spatial expression patterns of clock genes in the SCN are essentially similar for nocturnal and diurnal mammals (Challet, 2007), thus differential temporal expression of rhythms are most likely effected downstream from the circadian clock. Specifically, metabolism has been linked to phase shifts in locomotor activity, lower ambient temperatures and food shortage have been shown to induce a reduction in nocturnal activity and an increase in diurnal activity (Van der Vinne et al., 2014).

The results of the present study show less intraspecific variability than previous studies. Differenc30es in experimental designs such as sample selection, housing arrangements and data collecting methods may be responsible for variation. We selected very specific individuals for our investigation, i.e. adult breeding females and non-breeding females, fairly 
similar in size. Considering that we observed differences between our two groups, a random sample including individuals of both groups are therefore more likely to show higher variation. Also when activity of several animals housed together are recorded simultaneously (i.e. not individual animals), individual rhythms cannot be distinguished and larger variation will be perceived (Lovegrove et al., 1993).

Data recording methods may also have an influence on the perceived variability of activity rhythms. (Oosthuizen et al., 2003) used three different methods of recording, wheel-running activity showed the cleanest dataset, since animals may perform other activities when not in the wheel that will not be detected. In contrast, e-mitter recordings were more cluttered as all movements are recorded, some of which may be too small to be detected by passive infrared captors. Hence larger inter-individual variation was reported.

The collective results of the current and previous studies on the Damaraland mole-rat suggest clear entrainment of locomotor activity patterns to specific light cycles in the laboratory, these rhythms also free-run under constant conditions (Lovegrove et al., 1993; Oosthuizen et al., 2003), demonstrating the existence of a functional circadian clock.

Nevertheless, the subterranean niche is by definition devoid of light and the presence thereof is an unnatural occurrence for mole-rats. Indeed, telemetry data for locomotor activity in Damaraland mole-rats in their natural habitat indicates that individuals are equally active during the day and night (Lovegrove, 1988), suggesting that daily activity rhythms may not be prominent under natural conditions. Some measure of time keeping for subterranean species may be of value for species that breed seasonally, however Damaraland mole-rats can breed all year round. Photoperiod may thus not be such an important factor for the wellbeing and survival of this species. 


\section{Activity levels at different ambient temperatures}

Laboratory animals are frequently housed at ambient temperatures below their thermoneutral zones (TNZ) (Gaskill et al., 2009). Mild cold stress may alter experimental responses of animals, the activity levels of several small mammal species have been demonstrated to change at different ambient temperatures (Gaskill et al., 2009; Gordon, 1993).

In the present study, we observed differences in the total amount of locomotor activity of Damaraland mole-rats at all three ambient temperatures investigated. Damaraland mole-rats were found to be least active around $30{ }^{\circ} \mathrm{C}$ and most active at $25{ }^{\circ} \mathrm{C}$, while an intermediate level of activity was observed at $20^{\circ} \mathrm{C}$. The TNZ of Damaraland mole-rats range between 27$31{ }^{\circ} \mathrm{C}$ (Bennett, Clarke \& Jarvis, 1992; Lovegrove, 1986).

Several small laboratory rodent species prefer temperatures closer to their TZN for inactivity and maintenance behaviour, whereas cooler temperatures are selected as activity increases (Gaskill et al., 2009; Gordon, 1993). The locomotor activity in both rats and marmosets are depressed when ambient temperatures approach $30{ }^{\circ} \mathrm{C}$ compared to cooler temperatures (Finger, 1976; Palkova, Sigmund \& Erkert, 1999). Similarly Damaraland mole-rats are also much less active at $30^{\circ} \mathrm{C}$ compared to $25^{\circ} \mathrm{C}$ and $20^{\circ} \mathrm{C}$.

Ambient temperature has been shown to influence sleep and wakefulness in mammals, environmental temperatures that approach the TNZ of animals promote sleep, whereas lower temperatures impair it (Jhaveri, Trammell \& Toth, 2007; Kumar, Mallick \& Kumar, 2009; Thomas \& Kumar, 2000). The lower activity level of mole-rats at $30^{\circ} \mathrm{C}$ may reflect a larger proportion of their time being spent sleeping and resting.

Considering the evidence from other small mammal species, the dramatic increase in activity of the Damaraland mole-rats at $25{ }^{\circ} \mathrm{C}$ compared to $30{ }^{\circ} \mathrm{C}$ is expected, since when the ambient temperatures are lower than the TNZ range of a species, the animals implement either behavioural or physiological measures to maintain their body temperatures (Akin, 2011). 
At colder ambient temperatures, higher levels of locomotor activity may be advantageous as it produces heat to maintain body temperatures. Responses of small mammals to lower ambient temperatures appear to be varied, in some cases animals have been shown to increase their locomotor activity with a drop in ambient temperature (Ishii et al., 1996), whereas there is also evidence of the same species decreasing their locomotor activity at lower ambient temperatures (Vaanholt et al., 2007). Our results indicate that Damaraland mole-rats decrease their activity at $20{ }^{\circ} \mathrm{C}$ when compared to $25^{\circ} \mathrm{C}$. Damaraland mole-rats are highly social and in their natural environment, as well as in the laboratory when housed within their colonies will make use of social thermoregulation. Huddling has been shown to yield a significant reduction in energy in the Damaraland mole-rat as well as another mole-rat species, the Mashona mole-rat (Kotze, Bennett \& Scantlebury, 2008). Mole-rats may therefore inherently be more prone to attempt heat conservation by reducing the surface area for heat loss by huddling when they are in a group or curling up into a ball when housed singly instead of elevating activity levels.

Interestingly, the Damaraland mole-rat did not require a substantial reduction in the ambient temperature to show a decrease in locomotor activity. Although Damaraland mole-rats are fully furred and show homeothermic traits (Bennett et al., 1992), they are known to have lower resting metabolic rates and poorer thermoregulatory abilities than the less social molerat species (Bennett et al., 2000). This may explain the behavioural response to a relatively small reduction in ambient temperature.

\section{Activity in reproductive and non-reproductive females}

Although nocturnal activity was more prominent in both groups of mole-rats, nonreproductive females exhibited significantly more day-time activity compared to reproductive 
mole-rats. Nocturnal activity is comparable between the reproductive and non-reproductive animals at both $20^{\circ} \mathrm{C}$ and $25^{\circ} \mathrm{C}$ while non-reproductive animals are more active at $30{ }^{\circ} \mathrm{C}$.

The elevated diurnal activity of the non-reproductive females is unlikely to be stress related, since mild stress is generally associated with a reduction in locomotor activity (Gorka, Moryl \& Papp, 1996; Naqvi et al., 2012), and not an increase as is observed in this study.

Age is known to influence the amount of locomotor activity exhibited in animals (Duffy et al., 1997), such that older animals are less active than younger ones. Two problems arise with this hypothesis, firstly since the animals used in this study were wild trapped, it is impossible to know the exact age of the animals other than that they were adult. Breeding animals are the founders of the colony, therefore by default the oldest individuals in the colony. However, depending on the age of the colony, non-reproductive animals from one colony may in reality be older than queens from younger colonies. Secondly, breeding animals appear to attain a greater age than their non-breeding counterparts (Dammann et al., 2011), therefore they likely age slower and may physically appear younger than non-reproductive animals from the same colony.

An alternate explanation is that in Damaraland mole-rat colonies, non-breeding animals are responsible for foraging and the majority of the maintenance work in the tunnel system (Faulkes \& Bennett, 2001; Jarvis, 1981), therefore the higher level of activity in non-breeding animals is to be expected.

Reproductive and non-reproductive animals differ in the proportion of activity exhibited during the day at the different temperatures. While non-reproductive animals maintain day time activity at a relatively constant $35 \%$ at all three temperatures presented, reproductive animals shows the largest proportion of day time activity at $30{ }^{\circ} \mathrm{C}$ (about $21 \%$ ) which then progressively drops as ambient temperature decreases $\left(15 \%\right.$ at $25{ }^{\circ} \mathrm{C}, 10 \%$ at $\left.20{ }^{\circ} \mathrm{C}\right)$. Locomotor activity rhythms of non-reproductive Damaraland mole-rats appear to be more 
consistent over the range of temperatures than those of the reproductive animals. The work related division of labour in social mole-rat colonies (Bennett et al., 1988a; Jarvis, 1981) may be partly responsible for this inconsistency. Since breeding animals do not perform many burrow maintenance activities in the tunnel system, it may give them more opportunity to adapt their activity levels to ambient temperatures than non-reproductive animals that are responsible for tunnel maintenance and searching of food. Why non-reproductive animals are specifically more active during the day, remains to be elucidated.

The daily rhythm of locomotor activity appears to be relatively flexible in the Damaraland mole-rat, and respond to relatively small changes in ambient temperatures. Differences in daily activity between reproductive and non-reproductive animals may serve to emphasize the physiological and morphological castes in the Damaraland mole-rat.

\section{Acknowledgements}

We would like to thank Bobby and Ethel Reyneke for permission to collect animals on their property. We acknowledge the DST-NRF for funding the South African Research Chair of Mammalian Behavioural Ecology and Physiology to NCB.

\section{Reference list}

Akin, J. A. (2011) Homeostatic processes for thermoregulation. Nature Education Knowledge, 3, 7.

Barnett, M., N. C. Bennett, S. R. Telford \& J. U. M. Jarvis (2003) Foraging in the subterranean social Damaraland mole-rat, Cryptomys damarensis: an investigation into size-dependent geophyte utilization and foraging patterns. Canadian Journal of Zoology, 81, 743-752.

Bennett, N. C. (1994) Reproductive suppression in social Cryptomys damarensis colonies - a lifetime of socially-induced sterility in males and females (Rodentia: Bathyergidae). Journal of Zoology, London, 234, 25-39.

Bennett, N. C. (2009) African mole-rats (family Bathyergidae): models for studies in animal physiology. African Zoology, 44, 263-270.

Bennett, N. C., G. H. Aguilar, J. U. Jarvis \& C. G. Faulkes (1994) Thermoregulation in three species of Afrotropical subterranean mole-rats (Rodentia: Bathyergidae) from Zambia and Angola and scaling within the genus Cryptomys. Oecologia, 97, 222-227. 
Bennett, N. C., B. C. Clarke \& J. U. Jarvis (1992) A comparison of metabolic acclimation in two species of social mole-rats (Rodentia, Bathyergidae) in southern Africa. Journal of Arid Environments, 22, 189-198.

Bennett, N. C., F. P. D. Cotterill \& A. C. Spinks (1996) Thermoregulation in two populations of the Matabeleland mole-rat (Cryptomys hottentotus nimrodi) and remarks on the general thermoregulatory trends within the genus Cryptomys (Rodentia: Bathyergidae). Journal of Zoology, London, 239, 17-27.

Bennett, N. C. \& C. G. Faulkes (2000) African mole-rats: ecology and eusociality. Cambridge, UK: Cambridge University Press.

Bennett, N. C. \& J. U. Jarvis (1988a) The social structure and reproductive biology of colonies of the mole-rat, Cryptomys damarensis (Rodentia, Bathyergidae). Journal of Mammalogy, 69, 293302.

Bennett, N. C., J. U. Jarvis \& F. P. D. Cotterill (1993a) Poikilothermic traits and thermoregulation in the Afrotropical social subterranean Mashona mole-rat (Cryptomys hottentotus darlingi) (Rodentia: Bathyergidae). Journal of Zoology, London, 231, 179-186.

Bennett, N. C., J. U. Jarvis \& K. C. Davies (1988b) Daily and seasonal temperatures in the burrows of African rodent moles. S.Afr.J.Zool., 23, 189-195.

Bennett, N. C., J. U. Jarvis, C. G. Faulkes \& R. P. Millar (1993b) LH responses to single doses of exogenous GnRH by freshly captured Damaraland mole-rats, Cryptomys damarensis. Journal of Reproduction and Fertillity, 99, 81-86.

Blanchong, J. A., T. L. McElhinny, M. M. Mahoney \& L. Smale (1999) Nocturnal and dirunal rhythms in the unstriped nile rat, Arvicanthis niloticus. Journal of biological rhythms, 14, 364-377.

Bozinovic, F., M. J. Carter \& L. A. Ebensperger (2005) A test of the thermal-stress and the cost-ofburrowing hypotheses among populations of the subterranean rodent Spalacopus cyanus. Comparative biochemistry and physiology. Part A, Molecular \& integrative physiology, 140, 329-336.

Broekman, M., N. C. Bennett, C. R. Jackson \& M. Scantlebury (2006) Mole-rats from higher altitudes have greater thermoregulatory capabilities. Physiology \& behavior, 89, 750-754.

Challet, E. (2007) Minireview: Entrainment of the suprachiasmatic clockwork in diurnal and nocturnal mammals. Endocrinology, 148, 5648-5655.

Daan, S. \& C. S. Pittendrigh (1976) A functional analysis of circadian pacemakers in nocturnal rodents. Journal of comparative physiology A, 106, 267-290.

Dammann, P., R. Sumbera, C. Masmann, A. Scherag \& H. Burda (2011) Extended longevity of reproductives appears to be common in Fukomys mole-rats (Rodentia, Bathyergidae). Plos ONE, 6, e18757.

Duffy, P. H., R. J. Feuers, J. L. Pipkin, A. Turturro \& R. W. Hart (1997) Age and temperature related changes in behavioral and physiological performance in the Peromyscus leucopus mouse. Mechanisms of Ageing and Development, 95, 43-61.

Ellerman, J. R. (1956) The subterranean mammals of the world. Transactions of hte Royal society of South Africa, 35, 11-20.

Faulkes, C. G. \& N. C. Bennett (2001) Family values: group dynamics and social control of reproduction in African mole-rats. Trends in Ecology \& Evolution, 16, 184-190.

Finger, F. W. (1976) Relation of general activity in rats to environmental temperature. Percept Mot Skills, 43, 875-890.

Gaskill, B. N., S. A. Rohr, E. A. Pajor, J. R. Lucas \& J. P. Garner (2009) Some like it hot: Mouse temperature preferences in laboratory housing. Applied Animal Behaviour Science, 116, 279285.

Gordon, C. J. (1993) Twenty-four hour rhythms of selected ambient temperature in rat and hamster. Physiology \& behavior, 53, 257-263.

Gorka, Z., E. Moryl \& M. Papp (1996) Effect of chronic mild stress on circadian rhythms in the locomotor activity in rats. Pharmacol Biochem Behav, 54, 229-234. 
Hart, L., N. C. Bennett, B. Malpaux, C. T. Chimimba \& M. K. Oosthuizen (2004) The chronobiology of the Natal mole-rat, Cryptomys hottentotus natalensis. Physiology \& behavior, 82, 563-569.

Herbst, M. \& N. C. Bennett (2006) Burrow architecture and burrowing dynamics of the endangered Namaqua dune mole rat (Bathyergus janetta) (Rodentia: Bathyergidae). Journal of Zoology, 270, 420-428.

Herbst, M., J. U. M. Jarvis \& N. C. Bennett (2004) A field assessment of reproductive seasonality in the threatened wild Namaqua dune mole-rat (Bathyergus janetta). Journal of Zoology, 263, 259-268.

Ishii, K., M. Kuwahara, H. Tsubone \& S. Sugano (1996) The telemetric monitoring of heart rate, locomotor activity, and body temperature in mice and voles (Microtus arvalis) during ambient temperature changes. Laboratory animals, 30, 7-12.

Jarvis, J. U. \& J. B. Sale (1971) Burrowing and burrow patterns of East African mole-rats Tachyoryctes, Heliophobius and Heterocephalus. J.Zool.Lond,, 163, 451-479.

Jarvis, J. U. M. (1981) Eusociality in a mammal: cooperative breeding in naked mole-rat colonies. . Science, 212, 571-573.

Jhaveri, K. A., R. A. Trammell \& L. A. Toth (2007) Effect of environmental temperature on sleep, locomotor activity, core body temperature and immune responses of C57BL/6J mice. Brain, behavior, and immunity, 21, 975-987.

Johnson, P. G. \& I. Zucker (1983) Lability and diversity of circadian rhythms of cotton rats Sigmodon hispidus. Am J Physiol, 244, R338-R346.

Kotze, J., N. C. Bennett \& M. Scantlebury (2008) The energetics of huddling in two species of molerat (Rodentia: Bathyergidae). Physiology \& behavior, 93, 215-221.

Kumar, D., H. N. Mallick \& V. M. Kumar (2009) Ambient temperature that induces maximum sleep in rats. Physiology \& behavior, 98, 186-191.

Lovegrove, B. G. (1986) The metabolism of social subterranean rodents: adaptation to aridity. Oecologia, 69, 551-555.

Lovegrove, B. G. (1987) Thermoregulation in the subterranean rodent Georychus capensis (Rodentia: Bathyergidae). Physiol. Zool., 60, 174-180.

Lovegrove, B. G. (1988) Colony size and structure, activity patterns and foraging behaviour of a colony of the social mole-rat Cryptomys damarensis (Bathyergidae). J.Zool.Lond,, 216.

Lovegrove, B. G. (1989) The cost of burrowing by the social mole rats (Bathyergidae) Cryptomys damarensis and Heterocephalus glaber: The role of soil moisture. Physiological Zoology, 60, 449-469.

Lovegrove, B. G., G. Heldmaier \& T. Ruf (1993) Circadian activity rhythms in colonies of 'blind' molerats, Cryptomys damarensis (Bathyergidae). S.Afr.J.Zool., 28, 46-55.

Lovegrove, B. G. \& A. Knight-Eloff (1988) Soil and burrow temperatures, and the resource characteristics of the social mole-rat Cryptomys damarensis (Bathyergidae) in the Kalahari desert. Journal of Zoology, 216, 403-416.

Luna, F. \& C. D. Antinuchi (2007) Energetics and thermoregulation during digging in the rodent tucotuco (Ctenomys talarum). Comparative biochemistry and physiology. Part A, Molecular \& integrative physiology, 146, 559-564.

Marhold, S. \& A. Nagel (1995) The energetics of the common moler at Cryptomys, a subterranean eusocial rodent from Zambia. J. Comp. Physiol. B, 164, 636-645.

McNab, B. K. (1966) The metabolism of fossorial rodents: A study of convergence. Ecology, 47, 712733.

Molteno, A. J. \& N. C. Bennett (2000) Anovulation in non-reproductive female Damaraland mole-rats (Cryptomys damarensis). Journal of Reproduction and Fertillity, 119, 35-41.

Mrosovsky, N. \& S. Hattar (2005) Diurnal mice (Mus musculus) and other examples of temporal niche switching. Journal of comparative physiology. A, Neuroethology, sensory, neural, and behavioral physiology, 191, 1011-1024. 
Naqvi, F., S. Haider, Z. Batool, T. Perveen \& D. J. Haleem (2012) Sub-chronic exposure to noise affects locomotor activity and produces anxiogenic and depressive like behavior in rats. Pharmacological Reports, 64, 64-69.

Nevo, E. (1979) Adaptive convergence and divergence of subterranean mammals. Ann.Rev.Ecol.Syst., 10, 269-308.

Oosthuizen, M. K., H. M. Cooper \& N. C. Bennett (2003) Circadian rhythms of locomotor activity in solitary and social species of African mole-rats (family: Bathyergidae). Journal of biological rhythms, 18, 481-490.

Palkova, M., L. Sigmund \& H. G. Erkert (1999) Effect of ambient temperature on the circadian activity rhythm in common marmosets, Callithrix j. jacchus (primates). Chronobiology international, 16, 149-161.

Refinetti, R. (2006) Variability of diurnality in laboratory rodents. Journal of comparative physiology A, 192, 701-714.

Refinetti, R. (2008) The diversity of temporal niches in mammals. Biological Rhythm Research, 39, 173-192.

Roper, T. J., N. C. Bennett, L. Conradt \& A. J. Molteno (2001) Environmental conditions in burrows of two species of African mole-rat, Georychus capensis and Cryptomys damarensis. Journal of Zoology London, 254, 101-107.

Skinner, J. D. \& C. T. Chimimba (2005) The mammals of hte Southern African subregion. Cambridge, New York, Melbourne, Madrid, Cape Town, Singapore, Sao Paulo: Cambridge University Press.

Streicher, S., J. G. Boyles, M. K. Oosthuizen \& N. C. Bennett (2011) Body temperature patterns and rhythmicity in free-ranging subterranean Damaraland mole-rats, Fukomys damarensis. PLoS One, 6, e26346.

Šumbera, R., W. N. Chitaukali, M. Elichová, J. Kubová \& H. Burda (2004) Microclimatic stability in burrows of an Afrotropical solitary bathyergid rodent, the silvery mole-rat (Heliophobius argenteocinereus). Journal of Zoology, 263, 409-416.

Thomas, T. C. \& V. M. Kumar (2000) Effect of ambient temperature on sleep-wakefulness in normal and medial preoptic area lesioned rats. Sleep Res Online, 3, 141-145.

Vaanholt, L. M., T. Garland, Jr., S. Daan \& G. H. Visser (2007) Wheel-running activity and energy metabolism in relation to ambient temperature in mice selected for high wheel-running activity. J Comp Physiol B, 177, 109-118.

Van der Vinne, V., S. J. Riede, J. A. Gorter, W. G. Eijer, M. T. Sellix, M. Menaker, S. Daan, V. Pilorz \& R. A. Hut (2014) Cold and hunger induce diurnality in a nocturnal mammal. PNAS, 111, 1525615260.

Vasicek, C. A., M. K. Oosthuizen, H. M. Cooper \& N. C. Bennett (2005) Circadian rhythms of locomotor activity in the subterranean Mashona mole rat, Cryptomys darlingi. Physiology \& behavior, 84, 181-191.

Vleck, D. (1979) The energy cost of burrowing by the pocket gopher Thomomys bottae. Physiological Zoology, 52, 122-136.

Zelova, J., R. Sumbera, J. Okrouhlik \& H. Burda (2010) Cost of digging is determined by intrinsic factors rather than by substrate quality in two subterranean rodent species. Physiology \& behavior, 99, 54-58. 